COMUNICAÇÃO CIENTÍFICA

\title{
EFEITO DA INTENSIDADE DE PODA NA PRODUÇÃO E QUALIDADE DE FRUTOS DE PESSEGUEIRO ${ }^{1}$
}

\author{
MICHÉL ALDRIGHI GONÇALVES ${ }^{2}$, CARINE COCCO ${ }^{3}$, GERSON KLEINICK VIGNOLO ${ }^{4}$, \\ LUCIANO PICOLOTTO 5 , LUIS EDUARDO CORREAA ANTUNES ${ }^{6}$
}

RESUMO - O presente estudo teve como objetivo avaliar o comportamento vegetativo e produtivo de pessegueiros quando submetidos a variações na intensidade de desponte dos ramos durante a poda de frutificação. Foram utilizadas plantas de duas cultivares, Riograndense e Leonense, e três intensidades de poda, poda curta (retirada de $2 / 3$ do comprimento do ramo), poda longa (retirada de $1 / 3$ do ramo) e poda sem desponte (manutenção dos ramos íntegros). De acordo com os resultados, as plantas de 'Riograndense' apresentaram frutos com maior diâmetro, já as de 'Leonense' apresentaram maior percentual de frutificação efetiva (40,91\%), firmeza de polpa (7,70 kgf), sólidos solúveis ( $11,12{ }^{\circ}$ brix) e número médio de frutos por planta $(70,11)$. A poda denominada poda curta proporcionou maior diâmetro de frutos e maior volume de massa fresca retirada da poda em verde (3.193,75 g.planta $\left.{ }^{-1}\right)$. A interação 'Riograndense' e poda curta proporcionou maior percentual de frutos colhidos $(91,02 \%)$, maior massa média de frutos $(141,62 \mathrm{~g})$ e maior volume de massa fresca na poda de frutificação $\left(3.188,14\right.$ g.planta $\left.{ }^{-1}\right)$, já a maior produtividade foi obtida em plantas de 'Riograndense', que receberam poda sem desponte $\left(10,82\right.$ t.ha $\left.^{-1}\right)$. Os diferentes tipos de poda influenciam no desenvolvimento das plantas, afetando o número de flores e frutos. Nas condições em que o experimento foi conduzido, podemos concluir que características como frutificação efetiva, firmeza de polpa e sólidos solúveis não são alteradas pelos diferentes tipos de poda, já as variáveis relacionadas à produtividade estão intimamente relacionadas com as variações de intensidade de poda de ramos.

Termos para indexação: Prunus persica, frutificação, poda.

\section{THE EFFECT OF PRUNING INTENSITY IN THE PRODUCTION AND QUALITY OF PEACH FRUITS}

\begin{abstract}
The present study aimed to evaluate the vegetative and productive behavior of peach trees when subjected to variations in the intensity of lopping the branches during pruning. We used plants of two cultivars 'Riograndense' and 'Leonense', and three pruning intensities, heavy pruning (removal of $2 / 3$ of the length of the branches), pruning (removing $1 / 3$ of the length of the branches) without lopping and pruning (maintenance of the branches intact). The 'Riograndense' plants showed larger fruits in diameter, since 'Leonense' had a higher percentage of fruit set (40,91\%), greater firmness (7,70 kgf), soluble solids (11,12 brix) and more fruits per plant $(70,11)$. Pruning called heavy pruning provided greater fruit diameter and greater volume of fresh mass retreated in the summer pruning $\left(3,193.75\right.$ g.plant $\left.^{-1}\right)$. The interaction 'Riograndense' $x$ heavy pruning provide a greater percentage of fruits $(91.02 \%)$, higher fruit mass (141.62) and higher volume of fresh mass in pruning $\left(3,188.14\right.$ g.plant $\left.{ }^{-1}\right)$, now highest yield was obtained in 'Riograndense' plants received without lopping pruning $\left(10.82\right.$ t.ha $\left.^{-1}\right)$. The different types of pruning influence the development affecting the number of flowers and fruits of the plants. In the conditions of the experiment was conducted we conclude that characteristics such as effective fruit firmness and soluble solids, are not altered by different types of pruning, since the variables related to production are closely linked to variations of pruning branches intensity. Index terms: Prunus persica, fruiting, pruning.
\end{abstract}

\footnotetext{
'(Trabalho 225-13). Recebido em: 02-07-2013. Aceito para publicação em: 04-06-2014.

${ }^{2}$ Eng. Agr. Doutorando em Fruticultura de Clima Temperado, UFPel, Pelotas - RS, Brasil. Bolsista CAPES. E-mail: aldrighimichel@gmail.com ${ }^{3}$ Eng. Agr. Doutoranda em Fruticultura de Clima Temperado, UFPel, Pelotas - RS, Brasil. Bolsista CNPq. E-mail: carinecocco@ yahoo.com.br

${ }^{4}$ Eng. Agr. Doutorando em fruticultura de Clima Temperado, UFPel, Pelotas - RS, Brasil. Bolsista CAPES. E-mail: gerson_vignolo@ yahoo.com.br

${ }^{5}$ Eng. Agr. Dr. Bolsista PNPD/CAPES/Brasil. E-mail: picolotto@gmail.com

${ }^{6}$ Eng. Agr. Dr. Embrapa Clima Temperado, Pelotas - RS, Brasil. Bolsista CNPq, E-mail: luis.antunes@embrapa.br
} 
No Brasil, tanto a produção como a área cultivada com frutas de clima temperado têm crescido nos últimos anos; neste grupo, estão as frutas de caroço, nas quais predomina o pêssego, que em área cultivada representa mais de $80 \%$ (FACHINELLO et al., 2011). A região de Pelotas, no Rio Grande do Sul, destina mais de $90 \%$ de sua produção ao processamento industrial (TIMM et al., 2007), tendo a produção baseada em cultivares lançadas pelo programa de melhoramento genético da Embrapa Clima Temperado (RASEIRA et al., 2010).

Os produtores de pêssego do País adotam a prática cultural da poda de uma forma geral, independentemente da cultivar explorada, utilizando a mesma intensidade para os mais distintos genótipos, seguindo recomendações antigas que indicam o desponte dos ramos desenvolvidos no ano anterior, visando a estimular o desenvolvimento de novas brotações. É sabido que há a necessidade de adequar a intensidade da poda do pessegueiro aos distintos hábitos de crescimento, porta-enxertos, sistemas de condução e densidades de plantio (BASSI et al., 1994). Uma destas práticas amplamente adotada pelos produtores é o desponte de ramos durante a poda de frutificação, o que pode ocasionar respostas diferenciadas, dependendo das características de cada genótipo. Um fator importante para se definir o nível de desponte de ramos na poda de frutificação é o vigor de crescimento dos ramos. Dependendo deste vigor, pode ocorrer predominância de gemas vegetativas, principalmente na porção inferior dos ramos, sendo que algumas cultivares apresentam maior tendência a este comportamento.

A adoção generalizada da poda pode impossibilitar a expressão do verdadeiro potencial produtivo de algumas cultivares. Segundo Kumar et al. (2010), além da produtividade, a poda pode influenciar diretamente na qualidade do fruto, que no pêssego, assim como na maioria das frutas, refere-se a frutos grandes e firmes, com ótimo sabor, coloração, forma e textura. Contudo, o pêssego produzido na região Sul, embora de ótimo sabor, não raro deixa a desejar em tamanho, aparência e firmeza, quando comparado aos padrões internacionais (WAGNER JUNIOR et al., 2011). Desta forma, práticas de manejo do pomar e da planta são importantes ao alcance destes atributos de qualidade.

Além da qualidade do fruto, há também a necessidade de elevar a produtividade dos pomares, sendo que a baixa produtividade está diretamente relacionada a fatores como localização imprópria dos pomares, adubação desequilibrada, escolha equivocada de cultivares e manejo inadequado das plantas (BORGES et al., 2012). Por este motivo, antes de realizar a poda, deve-se conhecer o hábito de frutificação da espécie, assim como o comportamento da cultivar trabalhada quando submetido às variações de poda. Kumar et al. (2010) destacam que a mesma precisa ser adequada em termos de quantidade e intensidade, tendo em vista o comportamento da brotação e da frutificação das plantas manejadas.

Com o conhecimento das respostas da cultivar quando submetida a variações de intensidade de desponte de ramos, torna-se possível equilibrar as plantas do pomar de maneira mais lucrativa, estabelecendo um equilíbrio biológico entre os órgãos de crescimento, como os ramos, as folhas e as raízes. A poda do pessegueiro efetuada de forma desequilibrada influencia diretamente sobre a produção, podendo ocorrer a retirada de um número de gemas floríferas em excesso, ou favorecer um desequilíbrio, o que altera diretamente a capacidade fotossintética da planta (CHILDER; COWART, 1985).

Assim, objetivou-se, com a presente pesquisa, avaliar e caracterizar o comportamento de duas cultivares de pessegueiro sob os atributos vegetativos e produtivos quando submetidas a diferentes intensidades de poda de frutificação.

O experimento foi conduzido em uma área experimental pertencente à Embrapa Clima Temperado, localizada em Pelotas-RS, Brasil, coordenada $31^{\circ} 40$ ' 41,29 "S e $52^{\circ} 26^{\prime}$ '22,05" $\mathrm{W}$, altitude de $70 \mathrm{~m}$, sendo o mesmo avaliado no período de agosto a dezembro de 2010 , em um pomar instalado no ano de 2006 com duas cultivares tradicionalmente cultivadas na região de Pelotas-RS, 'Riograndense' e 'Leonense', ambas com exigência de aproximadamente 300 horas de frio para superar a dormência e florescer. $\mathrm{O}$ espaçamento utilizado foi de $1,5 \mathrm{~m}$ x $5 \mathrm{~m}$, e o sistema de condução adotado foi o Y duplo.

As combinações entre os fatores cultivar ('Riograndense' e 'Leonense') e intensidades de poda (poda curta (PC) com a retirada de $2 / 3$ do ramo produtivo, poda longa (PL) com a retirada de 1/3 do ramo, e poda sem desponte (PSD) com a manutenção dos ramos íntegros), sendo considerado o comprimento do ramo como base para definir os tratamentos, podendo existir assim uma pequena variação do número de gemas por ramo dentre os ramos do mesmo tratamento. Todos os tratamentos receberam uma poda-padrão no momento que receberam os tratamentos, que consiste na retirada de ramos débeis e ladrões (verticais e vigorosos), assim como uma poda em verde, aproximadamente 20 dias antes da colheita. A poda de frutificação ou poda na qual foram definidos os tratamentos foi realizada no 
estágio de inchamento de gemas.

As variáveis analisadas foram sólidos solúveis ( $\mathrm{SS}$ ) (em ` brix), determinados com o auxílio de um refratômetro digital com compensação de temperatura automática, de dez frutos por amostra; firmeza de polpa (Kgf), determinada, utilizando-se de um penetrômetro manual, com ponteira plana de $8 \mathrm{~mm}$ de diâmetro, a firmeza foi mensurada em dois pontos na região equatorial de cada fruto, sendo utilizados dez frutos por amostra; diâmetro de frutos ( $\mathrm{mm})$, obtido com auxílio de um paquímetro digital, colocado em posição perpendicular ao eixo do fruto, sendo realizadas duas medidas por fruto, em dez frutos por amostra; massa média de fruto $(\mathrm{g})$, determinada em balança digital com a pesagem de dez frutos por repetição.

Também foram avaliadas a frutificação efetiva (\%), obtida pela relação entre o número de frutos formados pelo número de flores abertas, de quatro ramos por planta, totalizando doze ramos por parcela, estabelecendo-se a porcentagem de frutificação por meio da expressão Fruit set $=\left(\mathrm{n}^{\circ}\right.$ de frutos $/ \mathrm{n}^{\circ}$ de flores) x 100; percentual (\%) de frutos colhidos, na colheita foi realizada a contagem dos frutos desenvolvidos nos ramos, onde anteriormente foi quantificada a frutificação efetiva; número de frutos por planta, obtido por meio da contagem do número total de frutos de cada planta; relação entre número de frutos pela seção transversal do tronco (fruto. $\mathrm{cm}^{-2}$ ), obtido por meio da divisão do número de frutos de cada planta pela área transversal do respectivo tronco; incremento do diâmetro do tronco (mm), obtido a partir da diferença entre as medidas realizadas individualmente do diâmetro do tronco de cada planta, no momento da prática da poda e após a colheita dos frutos; eficiência produtiva $\left(\mathrm{g} . \mathrm{cm}^{-2}\right)$, obtida pela relação entre a produção por planta e a área da seção do tronco; massa fresca da poda em seco e da poda em verde (g), obtida através da pesagem da massa fresca retirada de cada planta durante as distintas podas; produtividade estimada, considerando uma densidade de 1.333 plantas por hectare $\left(\mathrm{t} \cdot \mathrm{ha}^{-1}\right)$.

O delineamento experimental utilizado foi o de blocos casualizados, com quatro repetições, sendo utilizado um esquema fatorial em parcelas subdivididas, sendo considerado o fator cultivar na parcela (dois), e na subparcela, diferentes intensidades de poda (três). A unidade experimental foi composta de três plantas. Para todas as variáveis, foi procedida a análise de variância e, quando o efeito de tratamento foi significativo, realizou-se teste de comparação de médias (Tukey) $(\mathrm{p}<0,5)$.

O maior diâmetro médio de frutos foi registrado em 'Riograndense' (64,60 mm), diferindo estatisticamente de 'Leonense' (Tabela 1). Já as plantas submetidas à poda curta apresentaram frutos de maior diâmetro médio $(64,18 \mathrm{~mm})$, resultado este similar aos obtidos por Sharma e Chauhan (2004), na Itália, Kumar et al. (2010), na Índia, e Bussi et al. (2011), na França.

As plantas de 'Leonense' apresentaram frutos com maior firmeza média de polpa $7,7 \mathrm{kgf}$ (Tabela 1). Este comportamento pode ser explicado pelo menor diâmetro que os frutos desta cultivar quando comparados aos frutos de 'Riograndense,' concordando com Trevisan et al. (2006), que verificaram em Pelotas-RS, que frutos de pessegueiro com menor diâmetro apresentam maior firmeza de polpa. Assim como a firmeza de polpa, os frutos de 'Leonense' apresentaram média superior de sólidos solúveis $\left(11,12^{\circ}\right.$ brix $)$, já as intensidades de poda não influenciaram no teor de sólidos solúveis. Este resultado vai contra os resultados descritos por Sharma e Chauhan (2004), que verificaram em outras cultivares maiores teores de SS quanto maior a intensidade de poda aplicada. Já Bussi et al. (2011) descreveram que as distintas intensidades de poda não influenciam na concentração de sólidos solúveis dos frutos, sendo esta variável mais influenciada pela exposição à luz nas últimas semanas que antecedem a maturação dos frutos (GENARD; BRUCHOU, 1992).

A frutificação efetiva não sofreu efeito das diferentes intensidades de podas testadas, contrariando Citadin et al. (2008), que, estudando a frutificação efetiva de 'Granada', obtiveram resultados efetivamente positivos na frutificação com o uso de poda curta. Os mesmos autores afirmam que o efeito da poda curta seria devido à redução do número de gemas apicais dos ramos, o que proporciona menor competição por carboidratos entre as gemas remanescentes. No presente experimento, observou-se maior homogeneização da floração nas plantas que receberam poda curta e longa (dados não quantificados), comportamento provavelmente proporcionado pela redução da dormência correlativa exercida pelas gemas apicais. As plantas de 'Leonense' apresentaram maior percentual de frutificação efetiva $(40,91 \%)$, diferindo estatisticamente de 'Riograndense', que apresentou $17,15 \%$ (Tabela 1 ), resultado que comprova a baixa frutificação de 'Riograndense'.

Foi verificado que as plantas de 'Leonense' apresentaram maior número de frutos por área do tronco $\left(1,75\right.$ fruto.cm $\left.{ }^{-2}\right)$, assim como as podas sem desponte e longas (Tabela 1). A área da secção transversal do tronco é um índice utilizado por alguns 
produtores para definir o número aproximado de frutos que cada planta tem capacidade de suportar. Em pomares tradicionais, o índice utilizado é de cinco frutos por $\mathrm{cm}^{2}$ de tronco, e esse índice não pode ser transposto para o sistema de pomar compacto, pois levaria a um número excessivamente alto de frutos por planta, tornando-se necessário quantificar novos atributos baseados nas novas densidades de plantio e manejo dos pomares. No caso do presente estudo, esta variável foi utilizada na busca de diferenças motivadas pelos fatores estudados.

As plantas submetidas à poda curta apresentaram a menor eficiência produtiva entre os diferentes tipos de poda. Estes resultados demonstram que estas plantas poderiam ter melhor rendimento com poda mais longa dos ramos. Já o maior número de frutos foi registrado nas plantas de 'Leonense' $(70,11)$, assim como nas plantas que receberam a poda sem desponte $(74,91)$, não diferindo da poda longa (Tabela 1). Esta resposta é coerente, pois as plantas de 'Leonense' apresentaram melhor frutificação, independentemente do tratamento; por consequência, melhor desempenho produtivo; já a poda curta, por limitar o número de flores dos ramos, influenciou negativamente nesta variável.

As diferenças entre as cultivares não foram significativas para o incremento do diâmetro de tronco, sendo este influenciado apenas pelas distintas intensidades de poda. As plantas submetidas à poda sem desponte de ramos apresentaram a maior média $(9,11 \mathrm{~mm})$, comprovando uma relação inversa entre intensidade de poda e o incremento do diâmetro do tronco.

A massa fresca retirada na poda verde não diferiu entre as cultivares estudadas, já os níveis de poda apresentaram diferenças, sendo as plantas submetidas à poda curta as que apresentaram maior média de massa fresca $(3.193,75 \mathrm{~g})$, não diferindo estatisticamente das plantas submetidas à poda longa. As plantas que receberam a poda com maior intensidade apresentaram maior vigor nas brotações. Este comportamento pode ser explicado pelo fato de que, quanto maior a intensidade da poda, maior é o desequilíbrio entre parte aérea e raiz. Segundo DeJong et al. (2012), os ramos de pessegueiro geralmente apresentam inibição correlativa bastante forte, assim produzem poucos ramos secundários, e a poda pode afetar esta dominância, favorecendo o desenvolvimento de ramos oriundos de gemas basais. Bussi et al. (2011) relatam que podas severas proporcionam maior número de ramos indesejáveis na planta, sendo possível encontrar o ponto de equilíbrio da planta através da poda.

Para as variáveis percentuais de frutos colhidos, massa média de fruto, massa fresca de poda de frutificação e produtividade, foi verificada interação entre os fatores estudados. As plantas de 'Riograndense', que receberam poda curta, apresentaram os maiores percentuais de frutos colhidos $(91,02 \%)$, não diferindo estatisticamente de 'Leonense' com a mesma poda (Tabela 2). Já os menores percentuais foram verificados nas plantas que receberam a poda sem desponte dos ramos, em ambos os genótipos, mesmo que eles não tenham diferido da poda longa em 'Riograndense'. Os resultados demonstram uma relação inversa nos dois genótipos entre o percentual de frutos colhidos e o comprimento de ramos, podendo ser explicado pelo maior número de frutos nos ramos não despontados, resultando em maior concorrência por assimilados da planta.

As plantas de 'Riograndense' que receberam poda curta apresentaram frutos com maior massa média (141,62g) (Tabela 2). Mesmo as plantas de 'Leonense', apresentando frutos de menor massa média em todos os níveis de poda, quando comparadas com 'Riograndense', estas também apresentaram frutos de maior massa nas plantas submetidas à poda curta, resultados estes que concordam com os obtidos por Kumar et al. (2010) na Índia, onde verificaram que plantas submetidas a podas mais intensas apresentam frutos com maior massa média. Este resultado, provavelmente, tenha sido influenciado pelo menor número de frutos por plantas neste tratamento.

A interação entre 'Riograndense' e poda curta foi a que proporcionou maiores valores médios para a massa fresca da poda de frutificação (3.188,14 g.planta $\left.{ }^{-1}\right)$. Cabe salientar que, em todas as intensidades de poda, a cultivar Riograndense apresentou maiores médias para esta variável quando comparada com 'Leonense', evidenciando seu maior vigor. A maior produtividade foi obtida em plantas de 'Riograndense' que receberam poda sem desponte de ramos $\left(10,82\right.$ t.ha $\left.{ }^{-1}\right)$, não diferindo da interação entre a mesma cultivar com a poda longa (Tabela 2). Este resultado pode ser explicado pelas características intrínsecas desta cultivar que apresenta, em geral, maior massa média de frutos.

Já 'Leonense' teve maior produtividade quando comparada com 'Riograndense' apenas no tratamento com poda curta. A explicação para tal resultado está ligada à frutificação superior deste genótipo quando comparado com 'Riograndense'. Os menores valores para ambos os genótipos foram registrados nas plantas que receberam a poda curta, concordando com Kumar et al. (2010), que, testando diferentes intensidades de poda, verificaram que a 
produtividade diminui com o aumento da intensidade da poda.

Com o presente estudo, foi possível concluir que as características qualitativas, como firmeza de polpa e sólidos solúveis, não são alteradas pelas distintas intensidades de poda testadas. Já as diferentes podas influenciam diretamente nas variáveis relacionadas à produtividade, não sendo recomendada a poda curta para ambas as cultivares estudadas, principalmente para 'Riograndense', devido à sua reduzida frutificação efetiva.

TABELA 1 - Diâmetro de fruto, firmeza de polpa, sólidos solúveis (SS), Frutificação efetiva (FE), frutos por área de tronco $(\mathrm{FAT})$, eficiência produtiva $(\mathrm{EP})$, número de frutos por planta $(\mathrm{FP})$, incremento do diâmetro do tronco (IDT) e massa fresca poda em verde (MFPV) de pessegueiros submetidos a diferentes tipos de poda. Embrapa Clima Temperado, Pelotas-RS, 2011.

\begin{tabular}{lccc}
\hline \multicolumn{1}{c}{ Cultivares } & Diâmetro (mm) & Firmeza (Kgf) & SS ('brix) \\
\hline Riograndense & $64,60 \mathrm{a}^{*}$ & $6,17 \mathrm{~b}$ & $10,12 \mathrm{~b}$ \\
Leonense & $58,54 \mathrm{~b}$ & $7,70 \mathrm{a}$ & $11,12 \mathrm{a}$ \\
Tipo de poda & & & \\
Curta & $64,18 \mathrm{a}$ & $6,97 \mathrm{~ns}$ & $10,500^{\mathrm{ns}}$ \\
Longa & $59,43 \mathrm{~b}$ & 6,94 & 10,60 \\
Sem desponte & $61,10 \mathrm{~b}$ & 6,89 & 10,70 \\
$\mathbf{C V}(\mathbf{\%})$ & $\mathbf{3 , 0 6}$ & $\mathbf{7 , 4 8}$ & $\mathbf{6 , 6 1}$ \\
\hline
\end{tabular}

\begin{tabular}{|c|c|c|c|c|c|c|}
\hline Cultivares & $\begin{array}{l}\text { FE } \\
(\%)\end{array}$ & FAT (fruto.cm ${ }^{-2}$ ) & $\begin{array}{c}\mathrm{EP} \\
\left(\mathrm{g} \cdot \mathrm{cm}^{-2}\right)\end{array}$ & FP (fruto.planta ${ }^{-1}$ ) & IDT $(\mathrm{mm})$ & $\begin{array}{l}\text { MFPV } \\
(\text { g.pl-1) }\end{array}$ \\
\hline Riograndense & $17,15 \mathrm{~b}^{*}$ & $1,05 \mathrm{~b}$ & 132,69 ns & $53,77 \mathrm{~b}$ & $7,91^{\mathrm{ns}}$ & $2936,11 \mathrm{a}$ \\
\hline Leonense & $40,91 \mathrm{a}$ & $1,75 \mathrm{a}$ & 160,14 & $70,11 \mathrm{a}$ & 7,81 & $2798,61 \mathrm{a}$ \\
\hline \multicolumn{7}{|l|}{ Tipo de poda } \\
\hline Poda Curta & $30,77^{\mathrm{ns}}$ & $0,82 \mathrm{~b}$ & $104,18 \mathrm{~b}$ & $40,08 \mathrm{~b}$ & $6,83 \mathrm{c}$ & $3193,75 \mathrm{a}$ \\
\hline Poda Longa & 28,29 & $1,65 \mathrm{a}$ & $164,55 \mathrm{a}$ & $70,83 \mathrm{a}$ & $7,65 \mathrm{~b}$ & 2945,83 a \\
\hline Sem desponte & 28,04 & $1,72 \mathrm{a}$ & $170,51 \mathrm{a}$ & $74,91 \mathrm{a}$ & $9,11 \mathrm{a}$ & $2462,50 \mathrm{~b}$ \\
\hline $\mathrm{CV}(\%)$ & 28,24 & 42,82 & 42,06 & 12,96 & 8,82 & 22,96 \\
\hline
\end{tabular}

(*) Médias seguidas por letras minúsculas distintas, na mesma coluna, diferem entre si, pelo teste de Tukey, ao nível de $5 \%$ de probabilidade de erro, $\left({ }^{\text {ns }}\right)$ não significativo.

TABELA 2 - Percentual de frutos colhidos, massa média de fruto, massa fresca da poda de frutificação e produtividade de pessegueiros submetidos a diferentes tipos de poda. Embrapa Clima Temperado, Pelotas-RS, 2011.

\begin{tabular}{|c|c|c|c|}
\hline Cultivares & Poda curta & Poda longa & Sem desponte \\
\hline & \multicolumn{3}{|c|}{ Percentual de frutos colhidos $(\%)$} \\
\hline Riograndense & $91,02 \mathrm{Aa}^{*}$ & $82,41 \mathrm{Bb}$ & $80,24 \mathrm{Ab}$ \\
\hline Leonense & $86,86 \mathrm{Aab}$ & $88,66 \mathrm{Aa}$ & $81,43 \mathrm{Ab}$ \\
\hline \multirow[t]{2}{*}{$\mathrm{CV}(\%)$} & \multicolumn{3}{|c|}{8,30} \\
\hline & \multicolumn{3}{|c|}{ Massa média de frutos (g) } \\
\hline Riograndense & $141,62 \mathrm{Aa}$ & $125,29 \mathrm{Ab}$ & $121,59 \mathrm{Ab}$ \\
\hline Leonense & $119,61 \mathrm{Ba}$ & $90,46 \mathrm{Bb}$ & $76,84 \mathrm{Bb}$ \\
\hline \multirow{2}{*}{ CV(\%) } & \multicolumn{3}{|c|}{12,53} \\
\hline & \multicolumn{3}{|c|}{ Massa fresca da poda de frutificação (g.planta-1) } \\
\hline Riograndense & $3.188,14 \mathrm{Aa}$ & $2.775,51 \mathrm{Ab}$ & $1.995,75 \mathrm{Ac}$ \\
\hline Leonense & $2.724,53 \mathrm{Ba}$ & $2.403,05 \mathrm{Bb}$ & $1.742,49 \mathrm{Bc}$ \\
\hline \multirow[t]{2}{*}{ CV(\%) } & \multicolumn{3}{|c|}{21,1} \\
\hline & \multicolumn{3}{|c|}{ Produtividade (t.ha' $\left.{ }^{-1}\right)$} \\
\hline Riograndense & $5,72 \mathrm{Bb}$ & $10,67 \mathrm{Aa}$ & $10,82 \mathrm{Aa}$ \\
\hline Leonense & 7,94 Aa & $9,28 \mathrm{Ba}$ & $8,64 \mathrm{Ba}$ \\
\hline
\end{tabular}

(*) Médias seguidas por letras maiúsculas distintas, na mesma coluna, e médias seguidas por letras minúsculas distintas, na mesma linha, diferem entre si, pelo teste de Tukey, ao nível de 5\% de probabilidade de erro. 


\section{REFERÊNCIAS}

BASSI, D.; DIMA, A.; SCORZA, R. Tree structure and pruning response of six peach growth forms. Journal of the American Society for Horticultural Science, Mount Vernon, v.119, n.3, p.378-382, 1994.

BORGES, A. da F.; SILVEIRA, T.M.T.; SANTOS, J.; RASEIRA, M. do C.B. Tolerância de gemas floríferas, flores e frutos de pessegueiro a temperaturas de ocorrência de geadas. Revista Ceres, Viçosa, MG, v.59, n.3, p.355-359, 2012.

BUSSI, C.; BRUCHOU, C.; LESCOURRET, F. Response of watersprout growth to fruit load and intensity of dormant pruning in peach tree. Scientia Horticulturae, Amsterdam, v.130, n.4, p.725-731, 2011.

CHILDERS, N.F.; COWART, F.F. The photosynthesis, transpiration and stomata of apple leaves as affected by certain nutrient deficiencies. Journal of the American Society for Horticultural Science, Alexandria, v.33, p.160-163, 1985.

CITADIN, I.; LOCATELLI, M.C.; DANNER, M.A.; ASSMANN, A.P.; RASEIRA, M. do C.B.; NAVA, G.A. Floração, brotação e frutificação de pessegueiro cv. 'Granada' sob diferentes práticas de manejo. In: CONGRESSO BRASILEIRO DE FRUTICULTURA, 20., 2008, Vitória. Anais... CD-ROM.

DEJONG, T.M.; NEGRON, C.; FAVREAU, R.; COSTES, E.; LOPEZ, G.; GUÉDON, Y. Using Concepts of Shoot Growth and Architecture to Understand and Predict Responses of Peach Trees to Pruning. Acta Horticulturae, Wageningen, v.962, p.225-232, 2012.

FACHINELLO, J.C.; PASA, M.D.S.; SCHMTIZ, J.D.; BETEMPS, D.L. Situação e perspectivas da fruticultura de clima temperado no Brasil. Revista Brasileira de Fruticultura, v.33, n.1, p. 109-120. 2011. Número especial.
GENARD, M.; BRUCHOU, C. Multivariate analysis of within-tree factors accounting for the variation of peach fruit quality. Scientia Horticulturae, Amsterdam, v. 52, p.37-51, 1992.

KUMAR, M.; RAWAT, V.; RAWAT, J.M.S.; TOMAR, Y.K. Effect of pruning intensity on peach yield and fruit quality, Scientia Horticulturae, Amsterdam, v.125, n.3, p.218-221, 2010.

RASEIRA, M.doC.B.; NAKASU, B.H.; UENO, B.; SCARANARI, C. Pessegueiro: Cultivar BRS Kampai. Revista Brasileira de Fruticultura, Jaboticabal, v.32, n.4, p.1275-1278, 2010.

SHARMA, D.P.; CHAUHAN, J.S. Response of pruning intensities and fertilizer Treatments on yield, fruit quality and photosynthetic efficiency of peach, Acta Horticulturae, Wageningen, v.662, p.237-241, 2004.

TIMM, L. C.; REISSER JÚNIOR, C.; TAVARES, L.E.Q.; MADAIL, J.C.M.; MANKE, G.; LEMOS, F.D.; RADÜNZ, A.L.; LISBOA, H.; PRESTES, R.B.; MORO, M. Caracterização dos persicultores irrigantes e dos métodos de irrigação no polo produtivo de pêssego da região de Pelotas. Revista Brasileira de Agrociência, Pelotas, v.13, p.413-417,2007.

TREVISAN, R.; GONÇALVES, E.D.; CHAVARRIA, G.; ANTUNES, L E.C.; HERTER, F.G. Influência de práticas culturais na melhoria da qualidade de pêssegos. Revista Brasileira de Agrociência, Pelotas, v.12, n. 4, p.491-494, 2006.

WAGNER JÚNIOR, A.; BRUCKNER, C.H.; CANTÍN, C.M.; SÁNCHEZ, M.A.M.; CRUZ, C. D. Divergências genéticas entre progênies de pessegueiro em Zaragoza, Espanha. Revista Brasileira de Fruticultura, Jaboticabal, v.33, n.1, p.303-310, 2011. 